\title{
Correction to: Antecedents and outcomes of authentic leadership across culture: A meta-analytic review
}

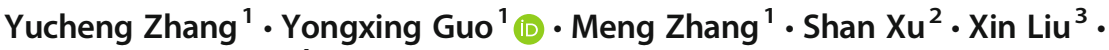 \\ Alexander Newman ${ }^{4}$ \\ Published online: 14 June 2021 \\ (C) Springer Science+Business Media, LLC, part of Springer Nature 2021
}

\section{Correction to: Asia Pacific Journal of Management https://doi.org/10.1007/s10490-021-09762-0}

Unfortunately, the Acknowledgement section was missing in the original version of this paper. Please see below:

Acknowledgements This research was funded by the National Natural Science Foundation of China (71972065, 71602163, 71701015) and General Program of Natural Science Foundation of Hebei Province (G2019202307).

The original article has been corrected.

The online version of the original article can be found at https://doi.org/10.1007/s10490-021-09762-0

\author{
Yongxing Guo \\ yongxing.guo@outlook.com \\ Yucheng Zhang \\ yucheng.eason.zhang@gmail.com \\ Meng Zhang \\ mengzhang_001@163.com \\ Shan $\mathrm{Xu}$ \\ sunshinexushan@163.com \\ Xin Liu \\ lliuxin@rmbs.ruc.edu.cn
}

Alexander Newman

a.newman@deakin.edu.au

Extended author information available on the last page of the article 
Publisher's note Springer Nature remains neutral with regard to jurisdictional claims in published maps and institutional affiliations.

\section{Affiliations}

\section{Yucheng Zhang ${ }^{1} \cdot$ Yongxing Guo ${ }^{1} \cdot$ Meng Zhang ${ }^{1} \cdot$ Shan $\mathrm{Xu}^{2} \cdot \mathrm{Xin}_{\mathrm{Liu}}{ }^{3} \cdot$ Alexander Newman ${ }^{4}$}

1 School of Economics and Management, Hebei University of Technology, Tianjin, China

2 School of Business Administration, Southwestern University of Finance and Economics, Chengdu, China

3 Renmin Business School, Renmin University of China, Beijing, China

4 Deakin Business School, Deakin University, Burwood, Australia 